\title{
Transition between direct and indirect band gap in silicon nanocrystals
}

\author{
V. Kocevski, O. Eriksson, and J. Rusz \\ Department of Physics and Astronomy, Uppsala University, Box 516, SE-751 20 Uppsala, Sweden \\ (Received 4 October 2012; revised manuscript received 20 February 2013; published 3 June 2013)
}

\begin{abstract}
Using ground-state density functional theory we study the transition from indirect to direct band gap in hydrogen-terminated silicon nanocrystals ( $\mathrm{NCs}$ ) as a function of decreasing diameter. The studied range, from 1.0 to $4.6 \mathrm{~nm}$ diameter of nanocrystals, with spherical and Wulff-shape NCs, covers the transition from nano- to bulk regime. A change in the symmetry of the lowest unoccupied state as a function of decreasing NC diameter is observed, gradually increasing the oscillator strength of transitions from the highest occupied to the lowest unoccupied state. Real space and Fourier space characteristics of highest occupied and lowest unoccupied states are explored in detail and linked to a smooth transition from nano- to bulk regime.
\end{abstract}

DOI: 10.1103/PhysRevB.87.245401

PACS number(s): 73.22.-f, 42.70.Qs, 61.46.Df, 78.40.Fy

\section{INTRODUCTION}

Silicon is among the most important materials in optoelectronic devices, such as photodetectors and photovoltaic cells. However, the use of bulk silicon, as an active material in light emitting devices, is limited because of its indirect nature of the electronic band gap. This disadvantage can be overcome by structural manipulation at the nanoscale. The discovery of photoluminescence from porous silicon ${ }^{1}$ confirmed that by confining the lateral dimensions silicon can emit light. Since then, a great interest had been devoted to the study of silicon nanocrystals, and their fundamental physical properties.

The nanocrystals (NCs) exploit effects of quantum confinement ${ }^{2,3}$-a change of the electronic structure of a system, when some of its dimensions become comparable to wavelengths of electrons in the sample. At these scales the band gap becomes wider and the absorption is blue-shifted, as the size is reduced. This increase of the Si band gap shifts it to the visible spectrum, and at the same time, the indirect nature of the gap undergoes a transformation. For an isolated nanocrystal there are no Bloch states and $\mathbf{k}$ vectors, since periodic boundary conditions are absent, therefore the notion of an indirect band gap has a different meaning. As already seen in previous works ${ }^{4-7}$ and also detailed below, the HOMO-LUMO gap (also called Kohn-Sham gap in literature) and the onset of optical absorption do in general occur at different energies, even in isolated NCs. This is the NC analog of the indirect band gap observed in bulk Si. Qualitatively, the widening of the gap can be understood as a narrowing of the bands, when the size of the system is reduced from bulk towards a single atom. As a consequence, if the center of the band remains approximately at the same energy, the distance between the edges of occupied and unoccupied bands becomes larger.

Semiempirically, the NCs are often modeled as a $\mathrm{Si}$ crystal with superimposed infinite spherical potential well ${ }^{8}$ employing the Luttinger model Hamiltonian ${ }^{9}$ and effective mass approximation. ${ }^{10}$ More recently, a number of investigations employed electronic structure calculations at various levels of sophistication, ranging from tight-binding methods, ${ }^{7,11,12}$ empirical pseudopotentials, ${ }^{13}$ via density functional theory ${ }^{4-6,14-16}$ (DFT), to the GW approximation combined with Bethe-Salpeter equation (BSE) or time-dependent DFT $^{17-20}$ and diffusion Monte Carlo calculations (DMC). ${ }^{21}$
These methods have generally confirmed the quantum confinement on an ab initio level and provided valuable insight into the electronic structure and optical properties, although, inevitably the GW, BSE, and DMC methods were limited to rather small Si clusters $(<1.5 \mathrm{~nm})$, so that details of the transition from nano- to bulk regime were not accessible.

In this study we turn our attention to the transition from the indirect band gap, observed in the bulk, towards a direct gap, seen in small NCs, and attempt to provide a microscopic explanation by means of ground-state DFT calculations of the electronic structure and absorption spectra. Although we perform only ground-state DFT calculations, it was found that the cancellation of many-body effects and the excitonic effects give rise to DFT HOMO-LUMO gaps comparable with the experimental optical gaps, ${ }^{5,12,21}$ although these effects go beyond the main interest of our study. Previously, Trani et al. ${ }^{7}$ used tight-binding methods to interpret this behavior of the band gap in Si NCs in terms of increasing localization of the highest occupied molecular orbital (HOMO) level in $\mathbf{k}$ space around the $\Gamma$ point, and localization of the lowest unoccupied molecular orbital (LUMO) level around the $\mathbf{k}$ point $\frac{2 \pi}{a}(0.83,0,0)$, as the diameter of the nanocrystal increases. Furthermore, Weissker et al. ${ }^{5}$ showed, using DFT-LDA, that with increasing size of the NCs, the tail in the energy dependence of oscillator strength is becoming longer, which has been assigned to development of bulklike properties. We extend these studies by a detailed investigation of HOMO and LUMO wave functions.

Structure of this manuscript is the following: In Sec. II we introduce our structural models for $\mathrm{Si} \mathrm{NCs}-$ a spherical model and two polyhedral structures with different planar terminations. We analyze the influence of NC shape on basic characteristics, such as cohesive energy, valence band width, and general shape of density of states. In Sec. III we discuss in detail the HOMO-LUMO and optical absorption gaps and their correlation with position-resolved electronic structure (surface or interior of NCs). In Sec. IV we inspect the spatial characteristics of highest occupied and lowest unoccupied eigenstates, HOMO and LUMO, both in real and reciprocal space. These provide an explicit picture of the gradual development of the bulk regime. Finally, concluding remarks are summarized in Sec. V. 


\section{STRUCTURAL MODELS OF SILICON NANOCRYSTALS}

We have analyzed the Si NCs of diameters $1.0 \mathrm{~nm}$ up to $4.6 \mathrm{~nm}$ using the pseudopotential DFT package SIESTA, ${ }^{22}$ which uses real space three-dimensional (3D) grid to appropriate the real space integrals, charge densities and potentials, and numerical atomic orbitals allowing for very efficient yet reliable calculations. The fineness of the grid is governed by the plane-wave cutoff. We have used plane-wave cutoff of $160 \mathrm{Ry}$, which gives converged energies and DOS for bulk silicon. The basis size for $\mathrm{Si}$ atoms was single $\zeta$ with polarization orbitals (SZP) and for hydrogen $(\mathrm{H})$ double $\zeta$. Since the main interest of our study are the Si NCs, and their properties, thus we consider that $\mathrm{H}$ does not take an active part in the studied properties. However, we have performed benchmark calculations for small Si clusters, using DZ and SZP for $\mathrm{H}$ and SZP for $\mathrm{Si}$, and we had seen that there is no significant difference in the band gaps for a particular cluster, when DZ or $\mathrm{SZP}$ basis is used for $\mathrm{H}$. In addition, the gain in computational efficiency, by excluding polarization functions, is significant, especially for the bigger NCs. Furthermore, the results of the SIESTA calculations with SZP and DZ basis sets for Si and $\mathrm{H}$, respectively, were cross-checked with the WIEN2K package, ${ }^{23}$ both in the bulk regime and for the smallest considered Si NC of diameter $1.0 \mathrm{~nm}$. Good agreement was observed for states up to $\sim 7.5 \mathrm{eV}$ above the Fermi level.

To see how the shape of the Si NCs affects the electronic properties, and whether there is any particular shape that the Si NCs would favor energetically, we tested three structural models - spherical and two types of polyhedral Wulff structures. $^{24}$ The spherical NCs are made by cutting out a sphere from bulk Si. The Wulff-shaped NCs are made by cutting along $\{110\}$ planes or $\{100\}$ and $\{111\}$ planes in bulk $\mathrm{Si}$. All Si atoms with only one Si neighbor were removed and the surface was passivated with hydrogen atoms. The volume of each structure is calculated as the volume of a convex hull drawn around the structure. The diameter of the NCs is calculated as a diameter of a sphere that has the same volume as the NCs' structure. Shown on Fig. 1 are the ball models of relaxed NCs. In Table I the diameters of relaxed NCs, and the number Si and $\mathrm{H}$ atom in the NCs are summarized.

The atom positions were relaxed by SIESTA for the whole range of $\mathrm{NC}$ shapes and sizes, until all forces on the atoms were lower than $0.04 \mathrm{eV} / \AA$. The importance of the structural relaxation for $\mathrm{Si} \mathrm{NCs}$ was pointed out in Ref. 6 for NCs of smaller sizes, demonstrating that an order of valence

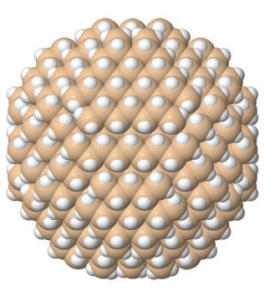

(a)

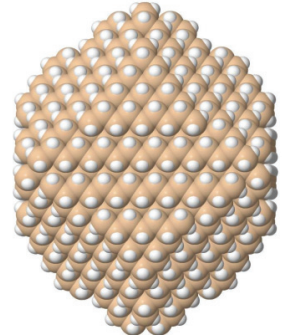

(b)

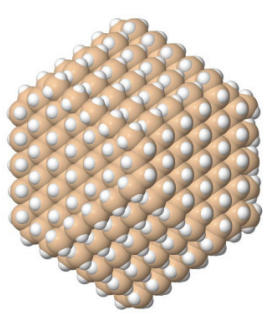

(c)
FIG. 1. (Color online) Ball models of relaxed Si NCs: (a) spherical, (b) Wulff $\{110\}$, and (c) Wulff $\{100\} /\{111\}$ structural models.
TABLE I. Diameter (in nanometers), and number of silicon and hydrogen atoms in Si NCs.

\begin{tabular}{|c|c|c|c|c|c|c|c|c|}
\hline \multirow{2}{*}{\multicolumn{3}{|c|}{$\begin{array}{l}\text { Spherical } \\
\text { structures }\end{array}$}} & \multicolumn{6}{|c|}{ Wulff structures } \\
\hline & & & \multicolumn{3}{|c|}{110} & $\multicolumn{3}{|c|}{100} /\{111\}$ \\
\hline$d(\mathrm{~nm})$ & $\mathrm{Si}$ & $\mathrm{H}$ & $d(\mathrm{~nm})$ & $\mathrm{Si}$ & $\mathrm{H}$ & $d(\mathrm{~nm})$ & $\mathrm{Si}$ & $\mathrm{H}$ \\
\hline 1.0 & 29 & 36 & & & & & & \\
\hline 1.5 & 87 & 76 & 1.5 & 87 & 76 & 1.1 & 35 & 36 \\
\hline 1.9 & 175 & 116 & 2.4 & 329 & 172 & 1.8 & 147 & 100 \\
\hline 2.5 & 389 & 196 & 2.8 & 555 & 252 & 2.5 & 377 & 196 \\
\hline 3.1 & 705 & 300 & 3.3 & 795 & 300 & 3.2 & 765 & 324 \\
\hline 3.5 & 1087 & 412 & 4.1 & 1213 & 412 & 3.8 & 1351 & 484 \\
\hline 4.2 & 1863 & 604 & 4.6 & 2320 & 660 & 4.6 & 2355 & 676 \\
\hline
\end{tabular}

and conduction states may change as a result of structural relaxation.

For each of the relaxed structures we have calculated normalized cohesive energies as a difference between the total energy of an $\mathrm{NC}$ and the sum of energies of free $\mathrm{Si}$ and $\mathrm{H}$ atoms, of which the NC consists. The cohesive energies were normalized per atom using the following equation:

$$
E_{\mathrm{c}}=\frac{E(\mathrm{NC})-\sum_{i} E_{i}(\text { free }) \cdot N_{i}}{N},
$$

where the summation is over every atom type $i, E(\mathrm{NC})$ is the total energy of the NC, $E_{i}$ (free) is the energy of a free atom, and $N_{i}$ and $N$ are the number of atoms of type $i$ and the total number of atoms, respectively. Differently from the bulk, in an $\mathrm{NC}$ there is surface on which the atoms have less than four silicon neighbors, which will cause lowering of the cohesive energy. This lowering of the cohesive energy is expected to depend on the surface area, as well as on the number of the fourfold coordinated $\mathrm{Si}$ atoms, i.e., it will depend on the surface to volume ratio. Having in mind that the NC's surface is not perfect, and there are differently coordinated silicon atoms on it, the cohesive energy is expected to follow a power-law function of the following form:

$$
E_{\mathrm{c}}(d)=E_{\mathrm{c}, 0}-\frac{A}{d^{B}},
$$

where $E_{\mathrm{c}}$ and $E_{\mathrm{c}, 0}$ are the normalized cohesive energy of the $\mathrm{NC}$ and of the bulk Si, both in $\mathrm{eV}, d$ is the diameter of the NC in $\mathrm{nm}$, and $A, B$ are parameters, which depend on the used exchange and correlation potential, LDA or GGA (the values for $A$ and $B$ are given in Fig. 2). Regardless of the shape of the NCs, Wulff-shaped or spherical NCs, all follow a universal function, with average accuracy of $\sim 0.35 \%$. This shows that when the cohesive energies are considered, any of the three structural models can be used as a model for Si NCs. However, our interest is in the changes in the band gaps with the size of the Si NCs, thus further analysis of the electronic properties of the NCs is needed to support the previous observation.

To see how the electronic properties of the Si NCs depend on the structural model and the size of the NCs, we have calculated the density of states (DOS) for all of the relaxed NCs. Figure 3 shows GGA calculations of the DOS of bulk silicon and silicon NCs with different shapes (spherical and Wulff structures), as a function of the diameter of the NCs. The DOS curves are aligned so that the top of the valence band (VB) 


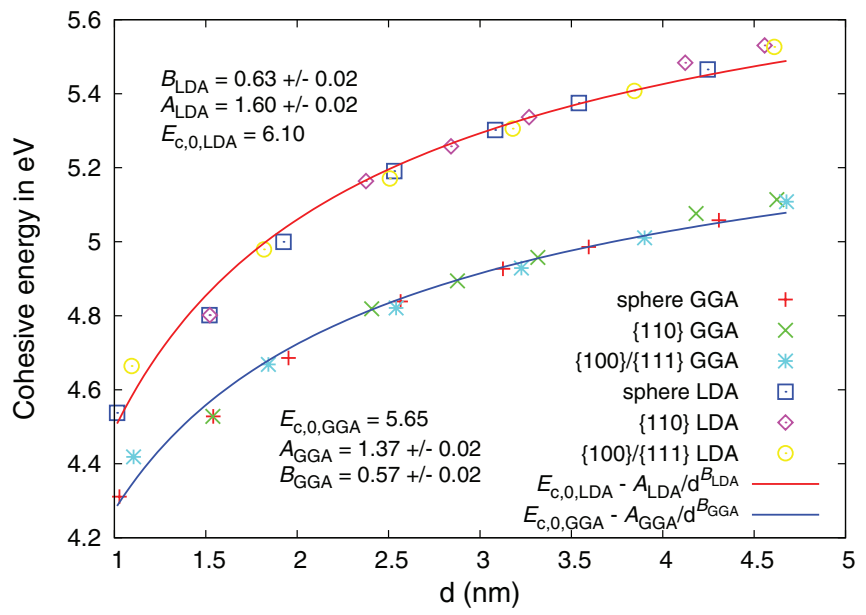

FIG. 2. (Color online) Comparison between the cohesive energies of Si NCs with different shapes (spherical and Wulff structures), calculated using LDA and GGA. The fitting curves, for the data calculated with LDA and GGA, is shown with solid red and blue lines, respectively.

is at $0 \mathrm{eV}$. Comparing the DOS of NCs with different shapes one can see that they are very similar to each other. A similar observation follows from the DOS calculated using LDA (data not shown).

As can be seen from Fig. 3, the width of the VB is getting smaller as the diameter of NC decreases. Following the quantum confinement effect, the narrowing of the VB causes

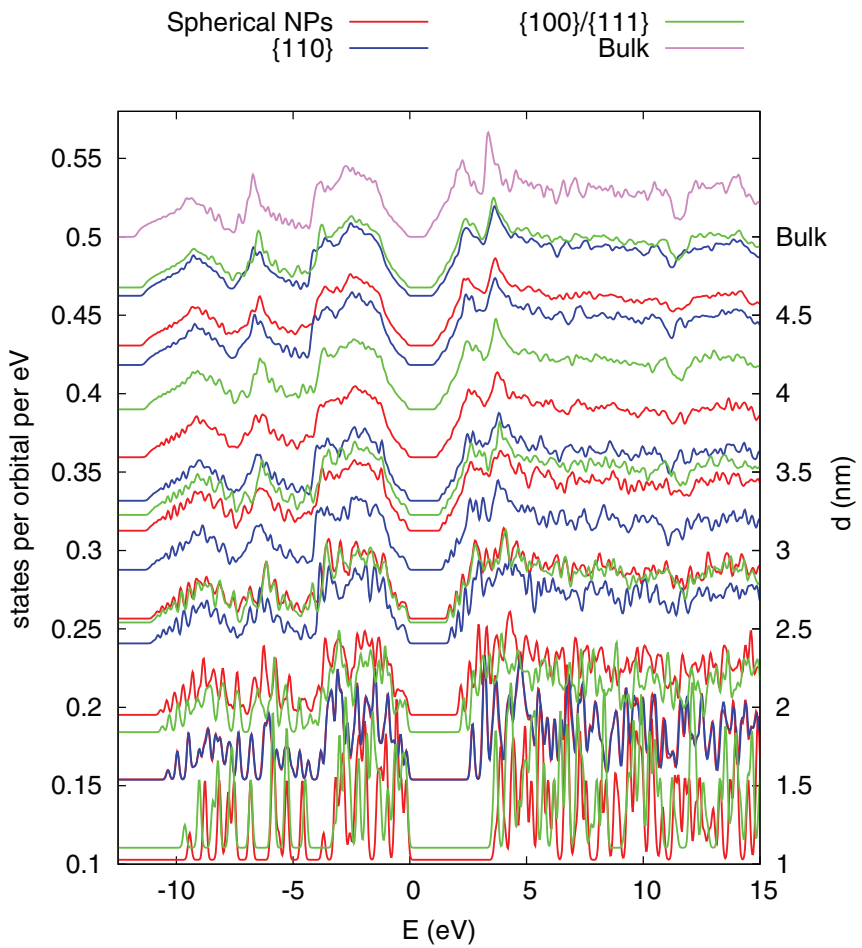

FIG. 3. (Color online) Density of states (DOS) of Si NCs with different shapes (spherical and Wulff structures), calculated using GGA. The DOS are shown as a function of diameter of the NC. The spherical, $\{110\}$ Wulff shapes and $\{100\} /\{111\}$ Wulff shapes are shown in blue, red, and green solid lines, respectively. The DOS of bulk silicon is shown in violet color as the top-most curve.

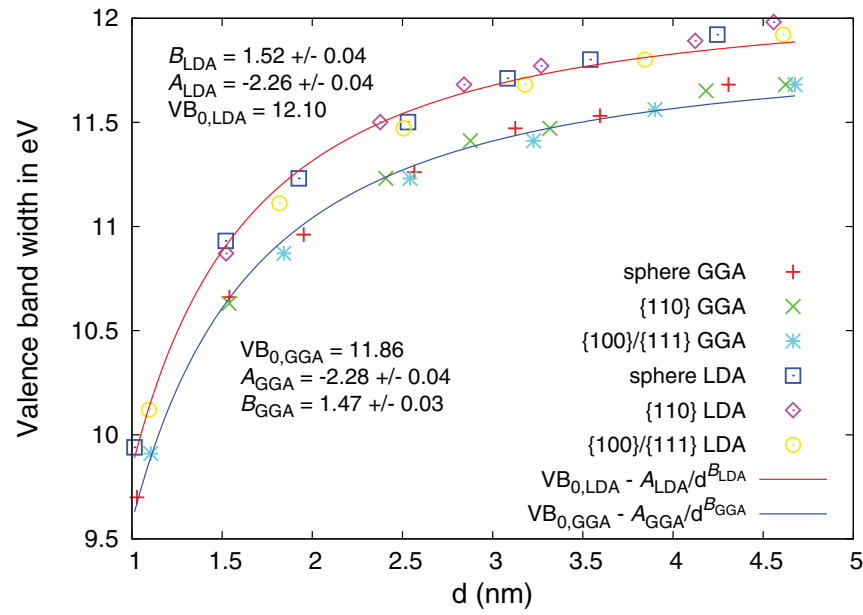

FIG. 4. (Color online) Valence band width of NCs with different shape (spherical and Wulff-shape structures), calculated using LDA and GGA. The fitting curves, for the data calculated with LDA and GGA, is shown with solid red and blue lines, respectively.

widening of the gap, ${ }^{25}$ which can also be seen on Fig. 3. To see if the dependence of the VB width is similar to the one of the HOMO-LUMO gaps, we have fitted the VB width using the same power-law function as in the case of band gaps reported by Delerue $e t a l .{ }^{11}$ - see the discussion about HOMO-LUMO gaps in Sec. III for more details. Figure 4 shows the VB width fit for both LDA and GGA, together with the valence band width of bulk $\mathrm{Si}\left(\mathrm{VB}_{0}\right)$ and the fit parameters $A, B$. It is worth noting that the cohesive energies calculated using LDA and GGA start to differ more for bigger NCs, whereas the difference in the VB widths calculated using LDA and GGA is almost the same throughout the studied NC diameters. This means that although LDA and GGA perform differently when total energies are in question, when it comes to the electronic properties, both exchange correlation potentials give similar results.

Comparing the size of the HOMO-LUMO gaps from NCs with different shapes, it is noticeable that they are very similar to each other. This observation, together with the correlation between the cohesive energies, and the similarities in the DOSes, shows that the spherical NCs are a suitable structural model of Si NCs. This is a good enough reason to use the spherical NCs in our further analysis of electronic structure of states around the band gap.

\section{FUNDAMENTAL AND OPTICAL ABSORPTION GAP}

Following the effective mass approximation the band gaps of Si NCs should follow a $d^{-2}$ law. Delerue et al. ${ }^{11}$ have shown that using a better description of the bands will cause lowering of the exponent, reporting a -1.39 exponent, which is getting bigger for bigger NCs, and eventually approaching 2 at infinitely big NCs. To see if our calculated HOMO-LUMO gaps, extracted from the DOS, show similar behavior, we have used the same power-law function to fit them. From our fits, see Fig. 5, it is evident that the dependence on the NC's diameter of the HOMO-LUMO band gap, is well represented by the powerlaw function, although with smaller exponent. Comparing the fitting curves for the HOMO-LUMO gaps and the VB width, 


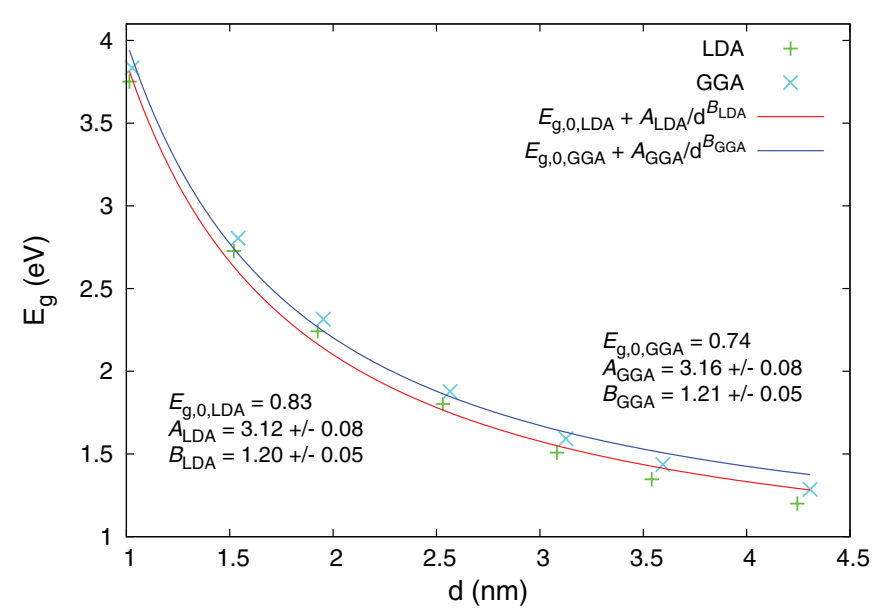

FIG. 5. (Color online) Comparison of the fitted fundamental gaps (defined in the text), for Si NCs with various diameters, calculated using different exchange correlation potentials: LDA (red) and GGA (blue).

it is noticeable that the HOMO-LUMO gaps are changing more rapidly with the diameter than the VB width, thanks to the larger magnitude of parameter $A$. This shows that the HOMO-LUMO gap does not depend solely on the VB width, but also on the changes in the conduction band width.

Here we should point out that in this work all the calculations of gaps are based on the ground-state density functional theory. Under the notion of fundamental gap we understand a difference between the energy of the lowest unoccupied eigenstate and the energy of the highest occupied eigenstate in an NC, which is also called the Kohn-Sham gap in the literature. ${ }^{5}$ Calculating the optical absorption gap on the base of ground-state DFT (also called an independent particle model) means that we employ a random-phase approximation (RPA) to the first-order time-dependent perturbation theory. ${ }^{26}$ For a detailed comparison to experiment one should include effects beyond the ground-state DFT, namely the many-body effects and the excitonic effects. ${ }^{27}$ It is interesting to note that more rigorous theoretical approaches have shown that excitonic and quasiparticle effects to a large extent cancel each other, and as a consequence, the HOMO-LUMO gaps compare well to measured optical gaps. ${ }^{5,12,21}$ Thus our comparison of theoretical and experimental results should be understood in this context. However, the main focus of our study is the direct vs indirect nature of optical transitions in terms of ground-state oscillator strengths, the nature of HOMO and LUMO states, and the evolution of these properties as a function of $\mathrm{NC}$ diameter. In this sense, the excitonic and many-particle effects go beyond the scope of our study.

To extract the optical absorption gap, we have calculated the imaginary part of the dielectric tensor by explicitly evaluating the dipole transition matrix elements between all occupied states and unoccupied states up to $15 \mathrm{eV}$ above the top of the valence band. Using the Kramers-Krönig transformation, the real part of the dielectric tensor was obtained, and hence the refraction and absorption indices-the latter is shown in Fig. 6. Our absorption spectra qualitatively agree with calculations of Ref. 13, although for smaller clusters our DFT calculations predict a broader spectrum than that of Ref. 13.

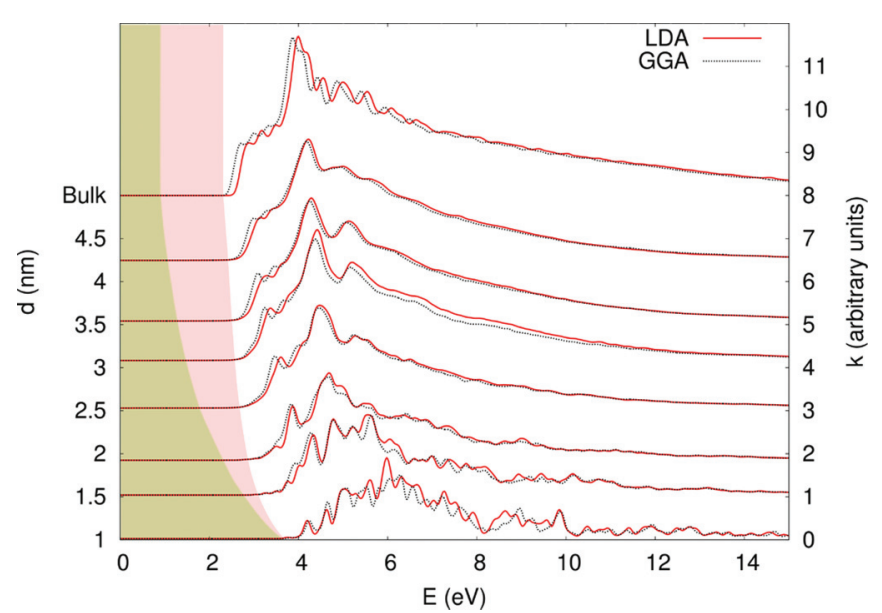

FIG. 6. (Color online) Absorption index (right axes) of Si NCs as a function of the diameter (left axes) and energy, and the exchange correlation potential: (i) LDA (red); (ii) GGA (dotted line). For bulk $\mathrm{Si}$ it is shown on the top (the $k$ values for the bulk are scaled by 2). The khaki and pink colored areas depict the HOMO-LUMO gap and the optical absorption gap, respectively.

The shapes of calculated absorption spectra agree well with previous measurements. ${ }^{29,30}$ A recent experimental work for 4-nm Si NCs ${ }^{31}$ also matches well with our calculations. From the absorption indices we extracted the optical absorption gap - an energy at which the absorption index becomes larger than a given threshold. Although, small nonzero contributions to the absorption index appear below this optical absorption threshold, these matrix elements are typically of negligible sizes. As a practical solution for the optical absorption threshold, we have chosen a value of 0.1 , which is two orders of magnitude smaller than the maximum of the absorption peak. Different threshold criteria have been used in literature (see, e.g., Ref. 7), all leading to very similar quantitative results.

A comparison of the fundamental gap and the optical absorption gap, extracted with the above-mentioned criterion, is shown in Fig. 7. As the size of the NC increases, the difference between the optical absorption and fundamental gap also increases, approaching the difference between the bulk $\Gamma-\Gamma$ gap and the bulk $\Gamma-X$ gap. Following the conception that bulk Si has an indirect gap, implying a difference between the optical absorption and fundamental gap, the coalescing of these two gaps at the smallest sizes, around $1.0 \mathrm{~nm}$, allows us to speak about $\mathrm{Si}$ in this size range as a direct gap material. This finding was previously obtained by tight-binding model calculations ${ }^{7}$ and was also confirmed by our WIEN2K calculations. For the largest considered NC both gaps are close to the bulk values. However, the fundamental and the optical absorption gaps of the bulk material are somewhat lower, suggesting an importance of the surface effects in all of the NC structures investigated here. Similar behavior of the fundamental and the optical absorption gaps has been observed in Si nanowires ${ }^{32,33}$ and nanotubes. ${ }^{34}$ There is a good qualitative agreement, in terms of trend, between the theoretical HOMO-LUMO gap and experimental data, ${ }^{35-38}$ as is clear from Fig. 7. This is in accord with above-mentioned cancellation of the quasiparticle and excitonic effects. However, there is noticeable difference between our calculated 


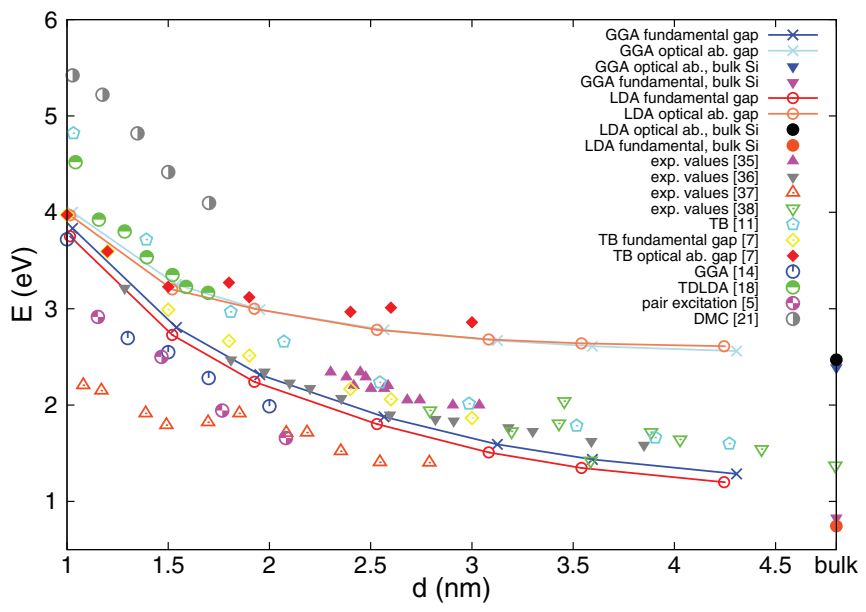

FIG. 7. (Color online) Comparison of fundamental and RPA optical absorption gap (as defined in the text) for Si NCs for various diameters, and different exchange correlation potentials, LDA and GGA, compared with data from published literature. $5,7,11,14,18,21,35-38$ Results obtained in this work are shown in red (LDA) and blue (GGA) colors, using dark (light) color for fundamental (absorption) band gaps, respectively.

HOMO-LUMO gaps and the experimentally observed gaps in Ref. 37. As interpreted by van Buuren et al. ${ }^{37}$ this difference can be attributed to the changes of the shape and the atoms on the surface of the experimentally analyzed NCs, whereas in calculations NCs with perfect spherical shape are used, terminated only with hydrogens.

For obtaining local electronic structure information, we have decomposed the DOS of the region around the gap (from the HOMO-LUMO gap to energies well above the optical absorption gap) layer-by-layer (see Fig. 8). The PDOSes around the gap of the surface layer, in each of the NCs, follow a convex curve (see Fig. 8, bottom right corner, for more details). It is noticeable that the number of states at the surface is smaller and is decreasing faster when approaching the gap, compared to the states in the interior of the NC. These features are already less pronounced in the layer right below the surface layer, which shows some similarities with the bulk, when comparing the height of the peaks from $s, p$, or $d$ states. In addition, for the layers below the surface the contribution from $s, p$, and $d$ states to the states around the gap, i.e., the relative height of the peaks from $s, p$, and $d$ states for a particular eigenstate, is similar to the contribution observed in the bulk. In the NC's interior (compared to the rest of the NC), the contribution to the states around the gap is bigger, which is evident from the intense peaks around the gap, particularly for smaller NCs. This shows that the states around the gap are localized in the NC's interior, implying that the lowest energy dipole transitions are localized there. Considering the similarity between the states of the layers below the surface and the bulk, and the indirect nature of the band gap in bulk $\mathrm{Si}$, it is expected that the intrinsic indirect nature of the Si will dominate in this region. Therefore, the dipole transitions in the region around the gap, which are not allowed in the bulk, should be suppressed or weak in this region. Indeed, it has been shown that the oscillator strength of these transitions is relatively small, and it is getting smaller as the size of the NC

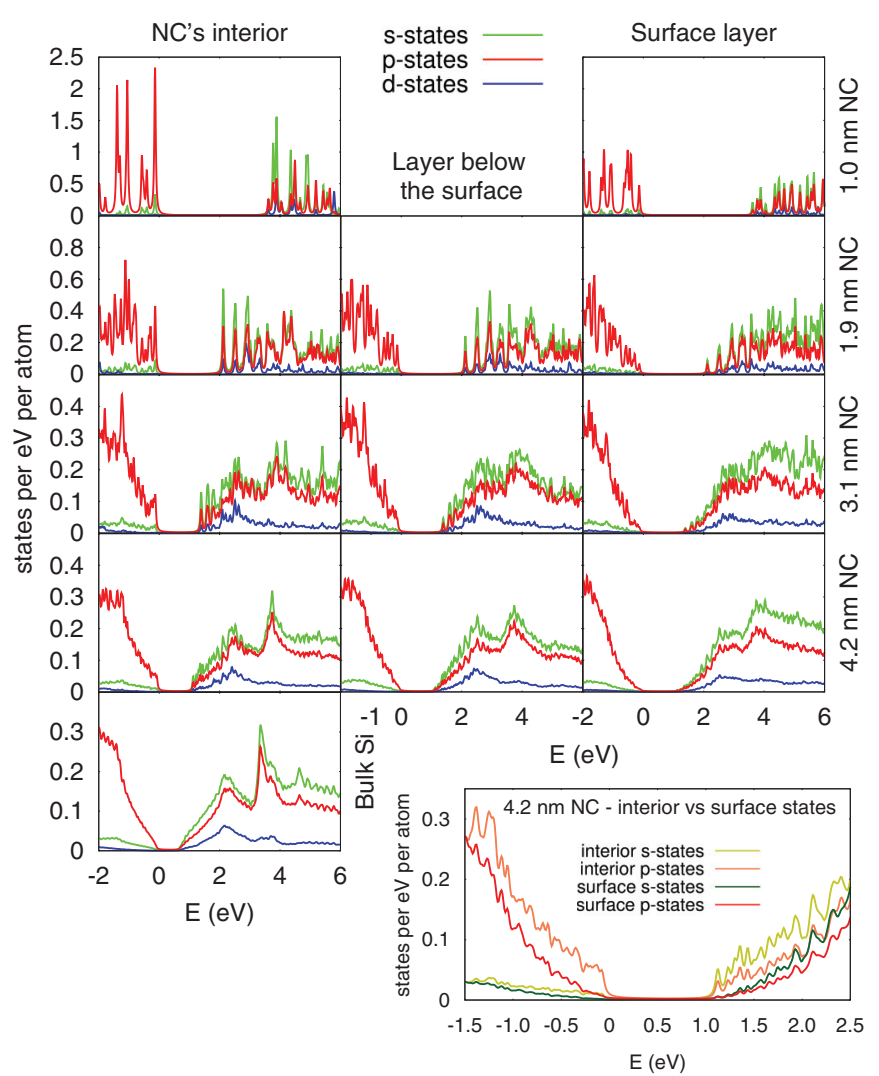

FIG. 8. (Color online) Projected density of states (PDOS) of Si NCs and of bulk Si. The $s, p$, and $d$ states are shown in green, red, and blue, respectively. The size of the NCs is shown on the right. The NC's interior PDOS is a sum over all the layers below the outer two layers. On the figure in the right bottom corner a comparison between the states in the interior and the surface of 4.2-nm NC are shown. The states in the interior are shown in lighter color, and the states in the surface in darker color.

is growing. ${ }^{5,7,28}$ In the small NCs, the states on the surface start to dominate, and these transitions become more probable. ${ }^{5,7,28}$ As the size of the NCs is growing, the difference between the optical absorption and fundamental gaps gets bigger. This picture is further supported by a study of the wave functions (WFs) around the gap.

\section{HIGHEST OCCUPIED AND LOWEST UNOCCUPIED EIGENSTATES}

According to DFT calculations, the HOMO is triply degenerate over the whole range of sizes, as in the nonrelativistic description of the Si bulk crystal. On the other hand, the degeneracy of the LUMO changes as a function of size: A nondegenerated state is found up to a diameter of $1.9 \mathrm{~nm}$, a doubledegenerated state is found at $2.5 \mathrm{~nm}$, and a triple-degenerated state for diameter of $3.1 \mathrm{~nm}$ and larger. In the following subsections we explore the NC size dependence of HOMO and LUMO wave functions both in real and reciprocal space.

\section{A. Real space characteristics}

In Fig. 9 we summarize the spatial characteristics of the HOMO and LUMO eigenstates. The first two rows display 

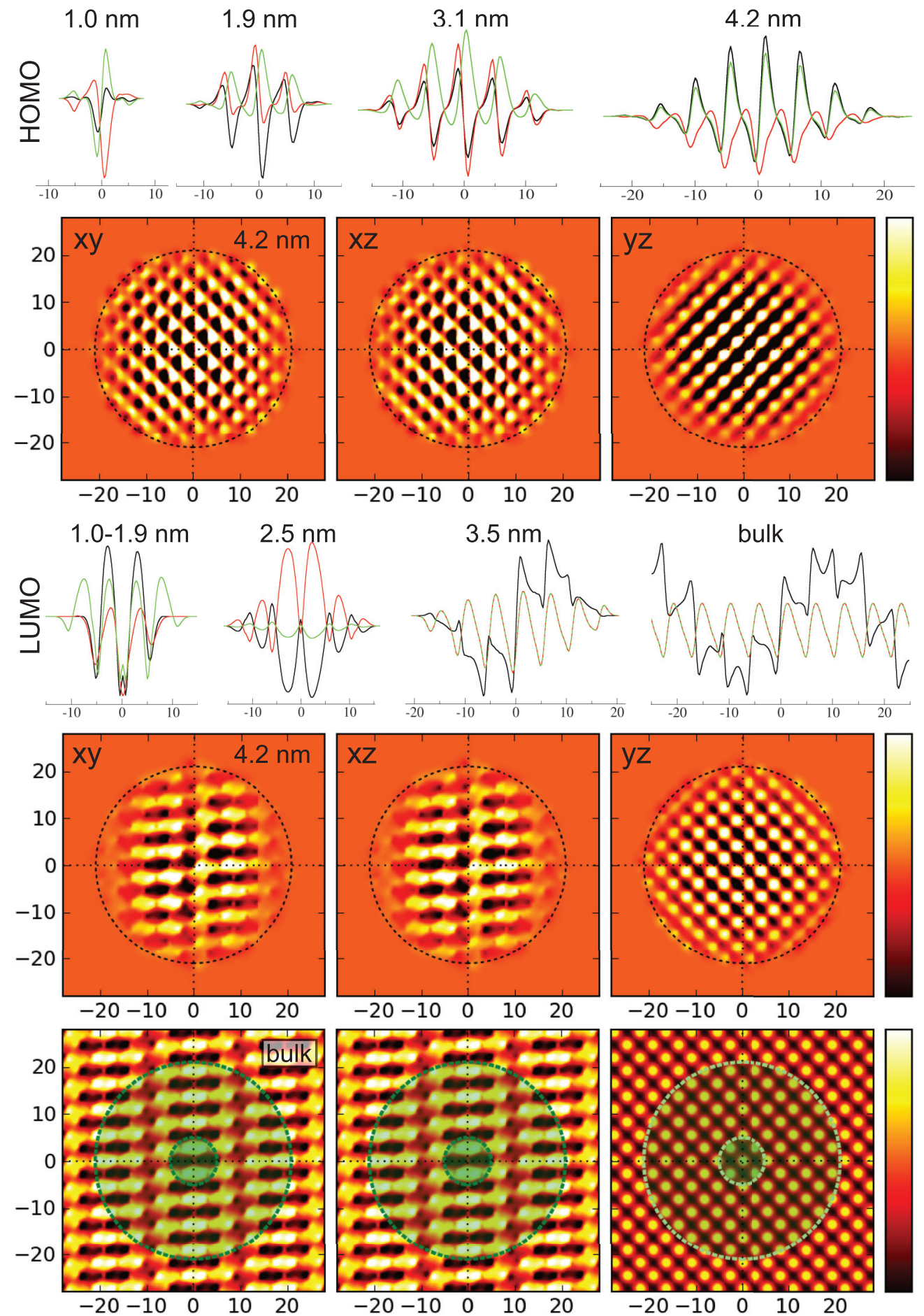

FIG. 9. (Color online) HOMO and LUMO wave functions of NCs of various sizes. The first row shows HOMO wave functions on a line passing through NC center parallel to lattice axes, for NCs of sizes 1.0, 2.0, 3.0, and $4.2 \mathrm{~nm}$, respectively. The second row shows three perpendicular planar cuts (parallel to lattice axes and passing through the center of NC) of the HOMO wave function for the 4.2-nm NC. The third row shows nondegenerate LUMO wave functions of 1.0-, 1.5-, and 1.9-nm NCs, respectively, then the three perpendicular cuts of LUMO for the 2.5-nm NC, the triple-degenerated LUMO of the 3.5-nm NC, and three perpendicular cuts of the bulk LUMO wave function. The planar cuts of LUMO in the 4.2-nm NC and bulk are compared in the fourth and fifth rows, respectively. All axes labeling is in $\AA$ units. The color bars are symmetric around zero, in arbitrary units.

the HOMO wave functions of NCs; the remaining part shows LUMO wave functions for NCs and bulk.

In the first row we plot the WF on a line passing along a lattice axis through the center of the NC. By construction there is always an atom in the center of our NC models. As mentioned above, HOMO is triple degenerated throughout the whole range of sizes. Therefore one can see three WFs, at each of the diameters, 1.0, 1.9, 3.1, and $4.2 \mathrm{~nm}$, respectively. 
Generally, these WFs have similar spatial characteristics across the whole range of NC sizes - an oscillatory behavior modulated with an envelope of a spherical Bessel function type ${ }^{8}$ causing the WF to decay towards the edges of the NC. These states have predominantly $p$ character, consistent with their threefold degeneracy (per spin). In the second line of Fig. 9 we show $x y, x z$, and $y z$ planar cuts of a HOMO eigenstate for the largest structural model. Due to the $p$-type symmetry of the HOMO wave function, the other two degenerated eigenstates would exhibit equal planar cuts, just with a cyclical interchange of the role of $x, y, z$ axes. These plots expectedly show $\Gamma$-type symmetry without any longer-range modulation, as would be the case of a developing Bloch state with nonzero $\mathbf{k}$ vector (see the next subsection).

The spatial characteristics of the LUMO eigenstates are rather different across the sizes of NCs. This was qualitatively interpreted by a higher number of Si-Si bonds, which leads to a $\mathbf{k}$-selection rule causing the dipole matrix element from HOMO to LUMO to be suppressed. ${ }^{28}$

For a 2.5-nm NC the nondegenerated state moves higher in energy, crossing two other eigenenergies. The LUMO becomes a double-degenerated state and the next lowest is a tripledegenerated state. The double-degenerated LUMO WF has an elliptically distorted $s$ symmetry-its WFs along the $x, y$, and $z$ directions, respectively, are all identical up to a sign and a scaling constant across both WFs. While one appears compressed along the $z$ direction, the other one is elongated along $z$. The linear plots along all three axes are shown in Fig. 9 in the third row.

NCs with diameter $3.1 \mathrm{~nm}$ or larger have a tripledegenerated LUMO of $p$-type symmetry. In Fig. 9, in the fourth row we show planar cuts of the LUMO. While the $y z$ plane shows a simple regular pattern with periodicity of the lattice parameter, the other two planar cuts of the LUMO WFs show mutually identical behavior with a longer wavelength modulation along the $x$ direction. This is indicative of a development of a Bloch state with wave vector $\mathbf{k}$ having a nonzero $x$ component. Note also the linear plots of the LUMO WF in the third row of Fig. 9, where LUMO of a 3.5-nm NC is compared to LUMO of the bulk Si. The similarity between the spatial characteristics of LUMO of larger NCs and bulk is clearly exposed by plotting the $x y, x z$, and $y z$ planar cuts of the LUMO WF for 4.2-nm NC (Fig. 9, fourth row) and bulk LUMO for $\mathbf{k}$ point $\frac{2 \pi}{a}(0.83,0,0)$ (Fig. 9, bottom row).

Oscillator strengths for transitions from HOMO to these states are negligible ${ }^{7,28}$ (or exactly zero in bulk), therefore the optical absorption and fundamental gaps differ. As we previously noted at the decomposition of the NC DOS layerby-layer (Fig. 8), the states around the gap are mostly localized in the interior of the NCs, which is consistent with the shape of the modulation of HOMO and LUMO cuts.

\section{B. K-space characteristics}

The development of the $\mathbf{k}$-selection rule between the HOMO and LUMO wave functions, as well as the gradual transition from a nanoregime to a bulk can be well elucidated in Fourier space. In Fig. 10 we are showing Fourier transforms of the HOMO and LUMO wave functions as a function of NC diameter from 1.0 to $4.2 \mathrm{~nm}$. The figures display the volume occupied by positive values of the real part of the Fourier transform, parallel projected to the $x z$ plane. The step size in the real space grid $\Delta x=0.267 \AA$ determines the dimension of the Fourier space as $\pm \frac{\pi}{\Delta x} \approx \pm 11.78 \AA^{-1}$.

For the NCs with smallest diameter the Fourier spectrum fills space without any signs of grid of spots, reminiscent of the periodic solid. But already at $1.9 \mathrm{~nm}$ we can observe a tendency of clustering of the maxima of the Fourier components, although these clusters are broad and overlap with their neighbor clusters.
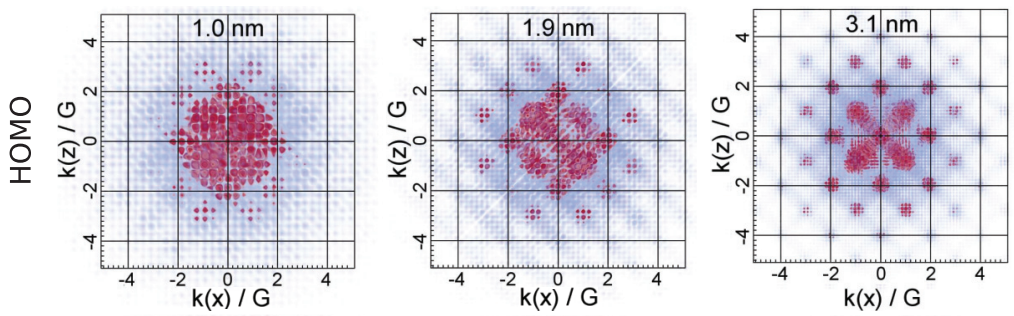

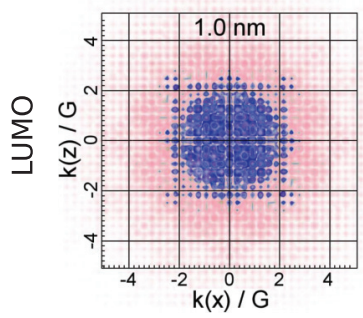

(a)

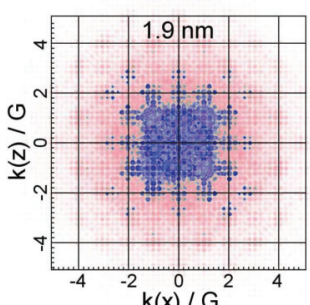

(b)

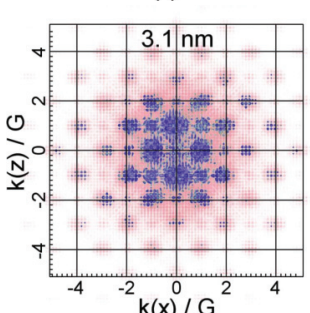

(c)

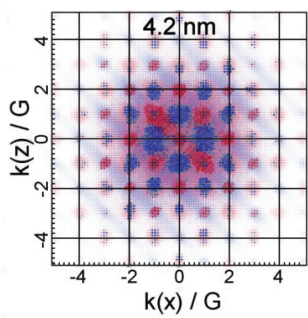

(d)

FIG. 10. (Color online) Fourier transform of the HOMO (upper row) and LUMO (lower row) wave function of Si NCs of the following sizes: (a) $1.0 \mathrm{~nm}$, (b) $1.9 \mathrm{~nm}$, and (c) $3.1 \mathrm{~nm}$. (d) Overlap between Fourier transform of the HOMO and LUMO wave functions for the 4.2-nm NC. The 3D Fourier transform was projected on the $x-z$ plane. For clarity of the graphical representation, the color range, blue to red for the HOMO WF and opposite, red to blue, for the LUMO WF, maps positive values of the real part of Fourier transform, within a fixed range for all sizes (in arbitrary units). The $\mathbf{k}(x)$ and $\mathbf{k}(z)$ are scaled by a reciprocal vector $\mathbf{G}$ of size $\frac{2 \pi}{a}$. 
At $3.1 \mathrm{~nm}$ the clustering of maxima of Fourier components of HOMO forms into an arrangement reminding a diffraction pattern of an fcc structure. Using the terminology of diffraction patterns, we see allowed reflections, which have Miller indices all odd or all even, and columns of forbidden reflections with low intensities, that have Miller indices $h$ and $l$ of different parity. It is very interesting to note that at this NC size there is a substantial qualitative difference between the Fourier transforms of HOMO and LUMO. While for HOMO we see a formation of fcc-like diffraction pattern, for LUMO - at first sight - it looks more like a diffraction pattern of a simple cubic structure with nonzero spots at all $h, k, l$ indices. On the other hand, a more detailed inspection reveals a different picture. Note that the spots corresponding to forbidden reflections are actually shifted towards the origin. We interpret this as an onset of the bandlike character of the LUMO wave function corresponding to the k point $\frac{2 \pi}{a}(0.83,0,0)$ and its degenerated symmetry-related counterparts.

At $4.2 \mathrm{~nm}$ the "Bragg spots" become even more sharp. Their shape and dimension is related to the Fourier transform of the complex hull of the NC models. At this NC size, HOMO shows a clear fcc-like pattern including a strong spot at the origin $(h k l)=(000)$. The Fourier transform of LUMO misses the central spot and although we can see weak spots corresponding to columns of Miller indices with $h= \pm 1$ and $l= \pm 1$, their intensity is much reduced compared to the grid originating from the $\mathbf{k}$ points corresponding to the LUMO of the bulk. This also shows that the two wave functions, HOMO and LUMO, developed almost mutually exclusive components in the Fourier space-as is the case of Bloch states in a bulk corresponding to different $\mathbf{k}$ points in the Brillouin zone. The mutually exclusive components of the 4.2-nm NC's HOMO and LUMO wave functions in Fourier space can be clearly see in Fig. 10(d), where the overlap of these two wave functions is shown.

\section{CONCLUSIONS}

In conclusion, using DFT calculations we have performed calculations of the electronic structure and optical properties of hydrogenated $\mathrm{Si} \mathrm{NCs}$, ranging from 1.0 to $4.6 \mathrm{~nm}$ in diameter, covering the transition from confined nanoregime to bulk regime. We have explored three types of structural models for NCs - a spherical model and two polyhedral Wulff-type structures-and found them equivalent in terms of cohesive energies and densities of states. We have argued that the narrowing of the valence band states contributes to the blue-shift of the band gap, as a function of reduced size of the NCs. For the sizes of the NCs, where a comparison is possible, we find good agreement between theory and experiment, regarding the trend of the fundamental band gap as a function of the NCs' size and optical absorption spectra. A detailed inspection of the HOMO and LUMO wave functions from the smallest sizes up to $4.2 \mathrm{~nm}$ has revealed a gradual onset of the bulk regime and development of the $\mathbf{k}$-selection rule.

\section{ACKNOWLEDGMENTS}

This work was supported by the EU's 7th Framework Programme SNAPSUN. J.R. and O.E. acknowledge the support of Swedish Research Council. O.E. also acknowledges support from the Knut and Alice Wallenberg foundation and the ERC (Project No. 247062-ASD), as well as STANDUPP and eSSENCE. The authors wish to thank an anonymous referee for his/her helpful comments on beyond-LDA effects.
${ }^{1}$ L. T. Canham, Appl. Phys. Lett. 57, 1046 (1990).

${ }^{2}$ D. J. Lockwood, Solid State Commun. 92, 101 (1994).

${ }^{3}$ A. I. Ekimov and A. A. Onushchenko, Sov. Phys. Semicond. 16, 775 (1982).

${ }^{4}$ S. Öğüt, J. R. Chelikowsky, and S. G. Louie, Phys. Rev. Lett. 79, 1770 (1997).

${ }^{5}$ H.-Ch. Weissker, J. Furthmüller, and F. Bechstedt, Phys. Rev. B 65 , 155328 (2002).

${ }^{6}$ H.-Ch. Weissker, J. Furthmüller, and F. Bechstedt, Phys. Rev. B 67, 245304 (2003).

${ }^{7}$ F. Trani, G. Cantele, D. Ninno, and G. Iadonisi, Phys. Rev. B 72, 075423 (2005).

${ }^{8}$ T. Takagahara and K. Takeda, Phys. Rev. B 46, 15578 (1992).

${ }^{9}$ J. M. Luttinger, Phys. Rev. 102, 1030 (1956).

${ }^{10}$ J. M. Luttinger and W. Kohn, Phys. Rev. 97, 869 (1955).

${ }^{11}$ C. Delerue, G. Allan, and M. Lannoo, Phys. Rev. B 48, 11024 (1993).

${ }^{12}$ C. Delerue, M. Lannoo, and G. Allan, Phys. Rev. Lett. 84, 2457 (2000).

${ }^{13}$ L.-W. Wang and A. Zunger, Phys. Rev. Lett. 73, 1039 (1994).

${ }^{14}$ Y. Wang, C. Chen, Z. Wu, W. Liang et al., Micro Nano Lett. 1, 1 (2011).

${ }^{15}$ E. Degoli, G. Cantele, E. Luppi, R. Magri, D. Ninno, O. Bisi, and S. Ossicini, Phys. Rev. B 69, 155411 (2004).
${ }^{16}$ J.-I. Iwata, A. Oshiyama, and K. Shiraishi, e-J. Surf. Sci. Nanotech. 8, 48 (2010).

${ }^{17}$ M. Rohlfing and S. G. Louie, Phys. Rev. Lett. 80, 3320 (1998).

${ }^{18}$ I. Vasiliev, S. Öğüt, and J. R. Chelikowsky, Phys. Rev. Lett. 86, 1813 (2001).

${ }^{19}$ L. X. Benedict, A. Puzder, A. J. Williamson, J. C. Grossman, G. Galli, J. E. Klepeis, J.-Y. Raty, and O. Pankratov, Phys. Rev. B 68, 085310 (2003).

${ }^{20}$ L. E. Ramos, J. Paier, G. Kresse, and F. Bechstedt, Phys. Rev. B 78, 195423 (2008)

${ }^{21}$ A. J. Williamson, J. C. Grossman, R. Q. Hood, A. Puzder, and G. Galli, Phys. Rev. Lett. 89, 196803 (2002).

${ }^{22}$ P. Ordejón, E. Artacho, and J. M. Soler, Phys. Rev. B 53, R10441 (1996); J. M. Soler, E. Artacho, J. D. Gale, A. García, J. Junquera, P. Ordejón, and D. Sánchez-Portal, J. Phys.: Condens. Matter 14, 2745 (2002).

${ }^{23}$ P. Blaha, K. Schwarz, G. K. H. Madsen, D. Kvasnicka, and J. Luitz, WIEN2K, An Augmented Plane Wave + Local Orbitals Program for Calculating Crystal Properties (Karlheinz Schwarz, Techn. Universität Wien, Austria, 2001).

${ }^{24} \mathrm{G}$. Wulff, Zeitschrift fur Krystallographie und Mineralogie 34, 449 (1901).

${ }^{25}$ L. Brus, J. Phys. Chem. 90, 2555 (1986); G. D. Stucky and J. E. Mac Dougall, Science 247, 669 (1990). 
${ }^{26}$ S. L. Adler, Phys. Rev. 126, 413 (1962).

${ }^{27}$ G. Onida, L. Reining, and A. Rubio, Rev. Mod. Phys. 74, 601 (2002).

${ }^{28}$ B. Delley and E. F. Steigmeier, Phys. Rev. B 47, 1397 (1993).

${ }^{29}$ N. Koshida, H. Koyama, Y. Suda, Y. Yamamoto, M. Araki, T. Saito, K. Sato, N. Sata, and S. Shin, Appl. Phys. Lett. 63, 2774 (1993).

${ }^{30}$ D. Amans, S. Callard, A. Gagnaire, J. Joseph, G. Ledoux, and F. Huisken, J. Appl. Phys. 93, 4173 (2003).

${ }^{31}$ M. Mansour, A. En Naciri, L. Johann, J. J. Grob, and M. Stchakovsky, Phys. Status Solidi A 205, 845 (2008).

${ }^{32}$ X. Zhao, C. M. Wei, L. Yang, and M. Y. Chou, Phys. Rev. Lett. 92, 236805 (2004).
${ }^{33}$ T. Vo, A. J. Williamson, and G. Galli, Phys. Rev. B 74, 045116 (2006).

${ }^{34}$ M. Ni, G. F. Luo, J. Lu et al., Nanotechnology 18, 505707 (2007).

${ }^{35}$ S. Furukawa and T. Miyasato, Phys. Rev. B 38, 5726 (1988).

${ }^{36}$ M. V. Wolkin, J. Jorne, P. M. Fauchet, G. Allan, and C. Delerue, Phys. Rev. Lett. 82, 197 (1999).

${ }^{37}$ T. van Buuren, L. N. Dinh, L. L. Chase, W. J. Siekhaus, and L. J. Terminello, Phys. Rev. Lett. 80, 3803 (1998).

${ }^{38}$ G. Ledoux, O. Guillois, D. Porterat, C. Reynaud, F. Huisken, B. Kohn, and V. Paillard, Phys. Rev. B 62, 15942 (2000). 